\title{
Multilevel Threshold Segmentation Method for Infrared Image of Power Equipment with Two-dimensional Entropy Based on Bat Algorithm
}

\author{
Xin $\mathrm{Li}^{\mathrm{a}}$, Haoyang Cui *b , Lunming Qin ${ }^{\mathrm{c}}$, Gaofang $\mathrm{Li}^{\mathrm{d}}$ \\ College of Electronics and Information Engineering, Shanghai University of Electric Power, \\ Shanghai, China \\ a e-mail: lixinshiep@126.com, ${ }^{\mathrm{b}}$ e-mail: cuihy@shiep.edu.cn, \\ ce-mail: lunming.qin@hotmail.com, ${ }^{\mathrm{d}}$ e-mail: lioneix_1128@sina.com, \\ *corresponding author
}

Keywords: power equipment; infrared image segmentation; bat algorithm; two-dimensional entropy

\begin{abstract}
The region of interest (ROI) segmentation of infrared image is one of the key steps in intelligent fault diagnosis of power equipment. Subsequently, a two-dimensional entropy multilevel threshold based on bat algorithm is proposed. By analyzing the multilevel threshold segmentation principle with two-dimensional entropy, the bat algorithm is used to search the optimal segmentation threshold, and these thresholds are used to the threshold segmentation experiment on the infrared image of the power equipment. The results show that this method improves time consuming of the algorithm and the precision of image segmentation compared with the multi-threshold segmentation method with two-dimensional entropy based on particle swarm optimization algorithm, which effectively solves the problem of multilevel threshold segmentation of infrared image. It lays the foundation for the extraction and analysis of temperature field characteristics of follow-up equipment, and is more suitable for the intelligent diagnosis of infrared images of power equipment failure.
\end{abstract}

\section{Introduction}

Infrared thermal imaging diagnostic technology has become one of the conventional means of fault diagnosis in power equipment ${ }^{[1-3]}$. The infrared (IR) image is effectively segmented and the temperature field of faulty device is extracted from the IR image. By analyzing the distribution of this field, we can determined the latent fault, the specific position of hidden trouble and the fault severity of the power equipment, quantitatively ${ }^{[4]}$.

The IR image of the power equipment is a pseudo-color image, which reflects the surface temperature distribution of power equipment with the characteristics of intensity concentration and low contrast. The traditional segmentation algorithm cannot distinguish the target from the background well. In addition, artificial given to the region of interest (ROI) reduced the efficiency of intelligent diagnostic system, obviously. Aiming at the difficulty of extracting region of IR image for power equipment, Hui Zou ${ }^{[5]}$ and others proposed a FAsT-Match algorithm which using the approximate affine transformation between visible and IR images. The approximate region of target in the IR image is obtained by the approximate position in the visible light, and then this segmentation algorithm obtains the segmentation result. Qiyin Wang ${ }^{[6]}$ proposed an adaptive IR image morphological segmentation method for power equipment of substation by using the K-means algorithm based on weighted Chebyshev distance. The above method can effectively solve the problem of threshold selection in the process of binarization of IR image, but merely extract the target device from the background. It cannot meet the requirement of IR temperature field feature analyzing and the fault diagnosis. Therefore, it is necessary to divide the IR image of the power equipment with multiple thresholds.

In order to solve this problem, this paper proposes a multilevel threshold segmentation method of two-dimensional entropy based on the bat algorithm, which can be used to analyze the temperature field of power equipment. The two-dimensional entropy formula is used as the fitness function of the bat algorithm to search for the optimal segmentation threshold of the IR image. These thresholds 
are used to divide the image into multiple thresholds. Compared with the multilevel threshold segmentation method of two-dimensional entropy based on particle swarm optimization (PSO) algorithm ${ }^{[7]}$, the proposed method is more accurate. It is more suitable for the extraction of temperature field, the feature analysis of power equipment and the intelligent diagnosis of fault subsequently.

\section{IR image segmentation with two-dimensional entropy method based on bat algorithm}

\subsection{Multilevel threshold segmentation method of two-dimensional entropy}

The size of temperature field image $I(x, y)$ is set to $M \times N$, and the range of gray scale is [0, $L-1]$. Assuming that the center pixel of a neighborhood is $(x, y)$. The average gray level of the $n \times n$ neighborhood is defined as $G(x, y)=\frac{1}{n^{2}} \sum_{i=-n / 2}^{n / 2} \sum_{j=-n / 2}^{n / 2} I(x+i, y+j)$. Let $f_{i, j}$ be number of the pixels. The gray value of the pixel is $i$ and gray value mean of the neighborhood is $j . P_{i, j}=f_{i, j} /(M \times N)$ is the probability of the pixel, and $\sum_{i=0}^{L-1} \sum_{j=0}^{L-1} p_{i, j}=1$. Fig. 1 shows a histogram of $x-y$ coordinate.

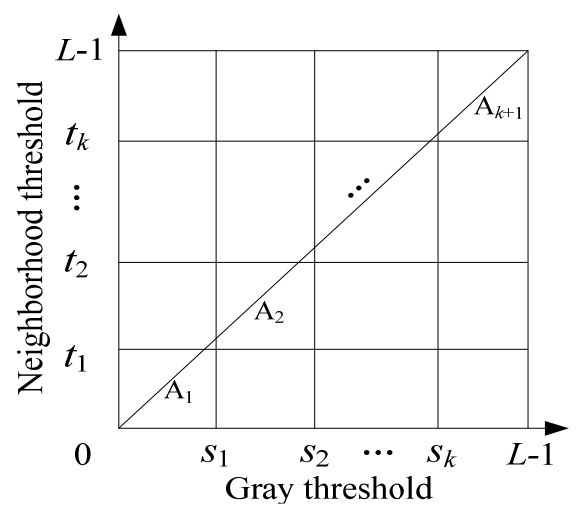

Fig.1 Two-dimensional multi-threshold histogram

$\mathrm{A}_{1}$ along the diagonal distribution represents the background area. $\mathrm{A}_{2}, \mathrm{~A}_{3}, \ldots, \mathrm{A}_{k+1}$ represent the target area respectively. The other areas away from the diagonal represent the boundaries and noise in the image. Therefore, the optimal thresholds should be determined on $\mathrm{A}_{1}, \mathrm{~A}_{2}, \ldots, \mathrm{A}_{k+1}$. Then we can use the two-dimensional maximum entropy method based on the gray value and gray scale mean value of the pixel, which can make the information of each region the largest. $p_{A_{1}}=\sum_{i=0}^{s_{1}-1} \sum_{j=0}^{t_{t}-1} p_{i, j}$ is the probability of threshold appears in $\mathrm{A}_{1}$, and the probability of other neighborhoods is equally available. Define the corresponding two-dimensional entropy of thresholds $\left(s_{1}, t_{1}\right),\left(s_{2}, t_{2}\right) \ldots\left(s_{k}, t_{k}\right)$ as ${ }^{[8]}$

$$
\begin{aligned}
& \Phi\left(s_{1}, t_{1} ; s_{2}, t_{2} ; \cdots ; s_{k}, t_{k}\right)=H_{1}+H_{2}+\cdots+H_{k} \\
& =-\sum_{i=a}^{s_{1}-1} \sum_{j=a}^{t_{1}-1}\left(\frac{p_{i, j}}{p_{A_{1}}}\right) \ln \left(\frac{p_{i, j}}{p_{A_{1}}}\right)-\sum_{i=s_{1}}^{s_{2}-1} \sum_{j=t_{1}}^{t_{2}-1}\left(\frac{p_{i, j}}{p_{A_{2}}}\right) \ln \left(\frac{p_{i, j}}{p_{A_{2}}}\right) \\
& -\cdots-\sum_{i=s_{k}}^{L-1} \sum_{j=t_{k}}^{L-1}\left(\frac{p_{i, j}}{p_{A_{k+1}}}\right) \ln \left(\frac{p_{i, j}}{p_{A_{k+1}}}\right) \\
& =\ln \left(p_{A_{1}} p_{A_{2}} \cdots p_{A_{k+1}}\right)-\frac{1}{p_{A_{1}}} \sum_{i=0}^{s_{1}-1} \sum_{j=0}^{t_{1}-1} p_{i, j} \ln \left(p_{i, j}\right)- \\
& \cdots-\frac{1}{p_{A_{k+1}}} \sum_{i=s_{k}}^{L-1} \sum_{j=t_{k}}^{L-1} p_{i, j} \ln \left(p_{i, j}\right)
\end{aligned}
$$

The discriminant function of entropy is defined as:

$$
\Phi\left(s_{1}{ }^{*}, t_{1}{ }^{*} ; s_{2}{ }^{*}, t_{2}{ }^{*} ; \ldots ; s_{k}{ }^{*}, t_{k}{ }^{*}\right)=\max \left\{\Phi\left(s_{1}, t_{1} ; s_{2}, t_{2} ; \ldots ; s_{k}, t_{k}\right)\right\}
$$


$\left(s_{1}{ }^{*}, t_{1}{ }^{*}\right)\left(s_{2}{ }^{*}, t_{2}{ }^{*} \cdots\left(s_{k}{ }^{*}, t_{k}^{*}\right)\right.$ are the thresholds.

\subsection{IR image segmentation with two-dimensional entropy based on bat algorithm}

In order to solve the problem of large computational complexity in the segmentation with two-dimensional entropy threshold, the bat algorithm is introduced. Thus, the thresholds selection problem in the multilevel threshold segmentation method is transformed into the optimization problem of the two-dimensional entropy function $\Phi\left(s_{1}{ }^{*}, t_{1}{ }^{*} ; s_{2}{ }^{*}, t_{2}{ }^{*} ; \ldots ; s_{k}{ }^{*}, t_{k}{ }^{*}\right)$ which is optimized by the bat algorithm.

Each bat in nature has a powerful echolocation ability to capture its prey by varying its frequency and loudness of sound waves during flight. In the bat algorithm, some idealized rules are used to simulate the process. The bat is randomly flying in space $x$ with the velocity $v$ and pulse frequency $f . f$ is adjusted by determining the degree of proximity to the prey. The optimization of the frequency is used to simulate the step size of the search, and the optimal solution in the optimization problem is simulated with the captured prey.

In the search space of $n t h$ dimension, the search frequency $f_{i}$, velocity $V_{i}^{t}$, and position $p_{t}^{i}$ of bat at time $t$ are represented as ${ }^{[9-11]}$ :

$$
\left\{\begin{array}{l}
f_{i}=f_{\text {min }}+\left(f_{\text {max }}-f_{\text {min }}\right) \beta \\
v_{i}^{t}=v_{i}^{t-1}+\left(p_{i}^{t-1}-p^{*}\right) f_{i} \\
p_{i}^{t}=p_{i}^{t-1}+v_{i}^{t}
\end{array}\right.
$$

Where $f_{\min }$ and $f_{\max }$ are the minimum and maximum search frequencies, respectively. $\beta$ is a random variable in the range $[0,1] \cdot p^{*}$ is current global optimal value. In the local search process, if a solution is selected from the optimal set, the location of the bat is updated to:

$$
p_{\text {new }}=p_{\text {old }}+\varepsilon A^{t}
$$

$\varepsilon$ is a random number in the range $[-1,1] . A^{t}$ is average loudness of all bats at time $t$. The balance between global and local optimization is achieved by changing loudness $A$ and pulse frequency $r_{i}$ :

$$
\left\{\begin{array}{l}
A_{i}^{t+1}=\xi A_{i}^{t} \\
r_{i}^{t+1}=r_{i}^{0}[1-\exp (-\theta t)]
\end{array}\right.
$$

Where $\xi$ is the loudness decreasing coefficient of $[0,1], \theta$ is the pulse frequency increase factor, $r_{i}^{0}$ indicates the maximum pulse frequency, $r_{i}^{t+1}$ is the pulse frequency at time $t+1$. The concrete steps are shown in Fig. 2. 


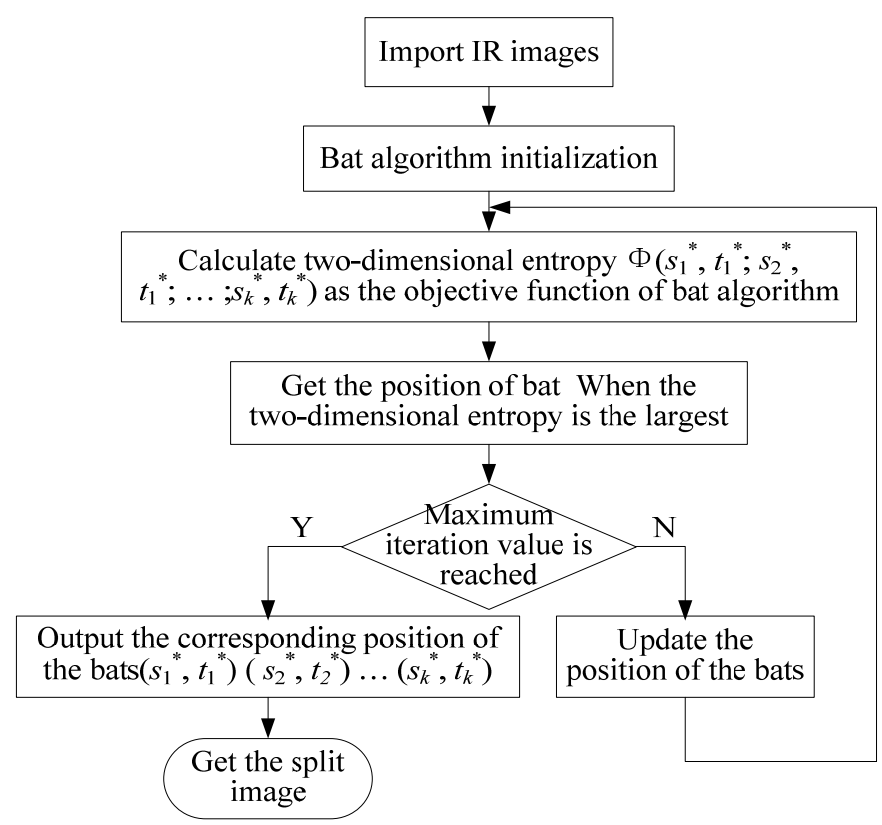

Fig.2 The flowchart of this algorithm

Through the bat algorithm, we can get the best segmentation threshold $t_{1}{ }^{*}$ and $t_{2}{ }^{*}, \ldots$, and $t_{k}{ }^{*}$, thus the IR image segmentation results of the equipment under $\mathrm{K}$ thresholds can be obtained.

\section{Experimental results and analysis}

In order to verify the segmentation effect of this method, we select the current transformer, the voltage transformer and the gate IR image as the experimental objects, and the images pixels are $640 \times 480$. Through the multilevel threshold segmentation experiment, we compare the proposed method with two-dimensional entropy threshold segmentation method based on PSO algorithm.

\subsection{IR image segmentation with multilevel threshold of two-dimensional entropy based on bat algorithm}

In this paper, a single-threshold, double-threshold, triple-threshold and quadruple-threshold segmentation method are performed for the three IR images of power equipment. The parameters in the experiment are set as follows: the maximum evolutionary algebra is 50, the pulse frequency range is $[0,1]$, the maximum pulse frequency $r=0.75$, the maximum pulse loudness $A=0.5$, the loudness decreasing coefficient $\xi=0.9$, the pulse frequency increasing coefficient $\theta=0.05$. The segmentation results are shown in Fig. 3. From top to bottom are the current transformer, voltage transformer and knife gate IR image. From left to right are single-threshold segmentation images, doubles, and triple and quadruple-threshold segmentation images, respectively. Single-threshold segmentation method extracted the hot region; double-threshold segmentation method extracts equipment and the hotspot area of equipment, simultaneously. Through triple-threshold and quadruple-threshold segmentation method, it makes a more detailed segmentation result of the IR temperature field image of the devices, and this method is suitable for the extraction and analysis of the temperature field characteristics of the equipment subsequently. 

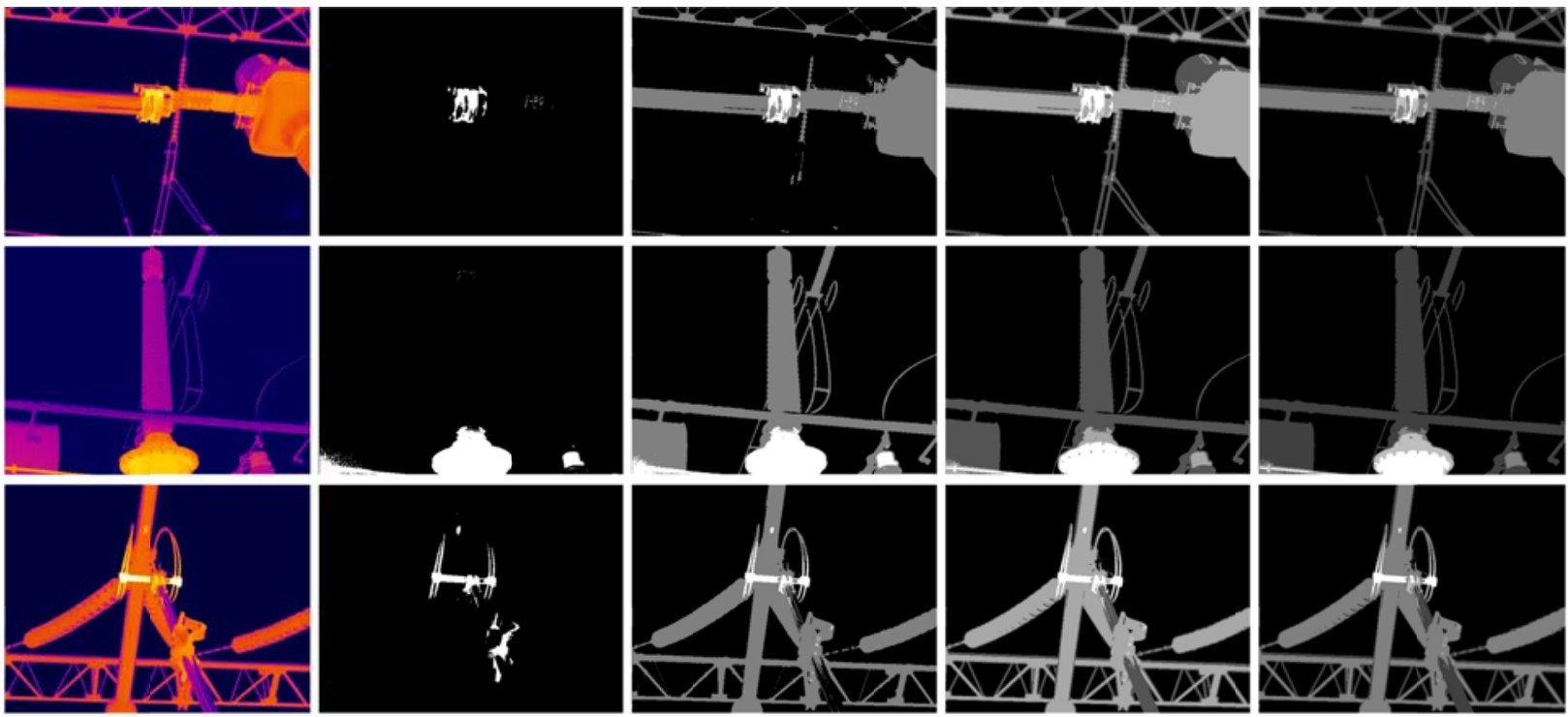

Fig.3 Experimental results of the proposed method

\subsection{Comparison experiment of multilevel threshold segmentation method with two-dimensional entropy based on PSO algorithm}

In order to evaluate the method, the SNR criterion is used to quantitatively analyze the performance of the algorithm. The SNR criterion is expressed as PSNR $=20 \log (255 / \mathrm{RMSE})(\mathrm{dB})$, where the RMSE value is as follows:

$$
\mathrm{RMSE}=\sqrt{\sum_{i=1}^{M} \sum_{j=1}^{N}(\mathrm{I}(i, j) \hat{\mathrm{I}}(i, j))^{2} / M N}
$$

$\mathrm{I}(x, y)$ and $\mathrm{I}(x, y)$ represent IR image and the segmented image with the pixel size of $M \times N$, respectively.

The PSO algorithm and the bat algorithm are used to optimize the two-dimensional entropy segmentation method, respectively. The gray-scale thresholds, time consuming and PSNR values of the two algorithms are shown in Table 1. It can be seen from table 1 that the time consuming of the algorithm in the single-threshold, double-threshold, triple-threshold and quadruple-threshold segmentation is decreased by $20.92 \%, 21.15 \%, 15.92 \%$ and $32.27 \%$, respectively. Moreover, the PSNR value of this method is lower than two-dimensional entropy segmentation method based on PSO algorithm. 
Table 1 Comparison of gray threshold, computation time and PSNR value of the two methods

\begin{tabular}{|c|c|c|c|c|c|c|c|}
\hline \multirow[b]{2}{*}{ Image } & \multirow{2}{*}{$\begin{array}{l}\text { Number of } \\
\text { thresholds }\end{array}$} & \multicolumn{3}{|c|}{ The method based on PSO algorithm } & \multicolumn{3}{|c|}{ The proposed method } \\
\hline & & $\begin{array}{c}\text { Gray } \\
\text { thresholds }\end{array}$ & $\begin{array}{c}\text { Time } \\
\text { consuming } / \mathrm{s}\end{array}$ & $\mathrm{PSNR} / \mathrm{dB}$ & $\begin{array}{c}\text { Gray } \\
\text { thresholds }\end{array}$ & $\begin{array}{c}\text { Time } \\
\text { consuming/s }\end{array}$ & $\mathrm{PSNR} / \mathrm{dB}$ \\
\hline \multirow{4}{*}{$\begin{array}{l}\text { IR image } \\
\text { of voltage } \\
\text { transformer }\end{array}$} & 1 & 165 & 0.6676 & 27.8412 & 165 & 0.5456 & 27.8412 \\
\hline & 2 & 81,165 & 0.7569 & 29.3414 & 81,165 & 0.5908 & 29.3414 \\
\hline & 3 & $21,98,165$ & 0.7778 & 32.1344 & $21,98,165$ & 0.6625 & 32.1344 \\
\hline & 4 & $17,75,145,180$ & 1.2215 & 29.5960 & $21,99,163,195$ & 0.8292 & 30.3888 \\
\hline \multirow{4}{*}{$\begin{array}{l}\text { IR image } \\
\text { of current } \\
\text { transformer }\end{array}$} & 1 & 82 & 0.6765 & 27.0647 & 82 & 0.5382 & 27.0647 \\
\hline & 2 & 83,151 & 0.7378 & 25.1433 & 19,83 & 0.5876 & 27.0243 \\
\hline & 3 & $19,83,173$ & 0.7969 & 26.9962 & $19,84,151$ & 0.6648 & 29.2089 \\
\hline & 4 & $19,84,132,173$ & 1.2368 & 28.8309 & $18,84,132,170$ & 0.8762 & 28.9104 \\
\hline \multirow{4}{*}{$\begin{array}{l}\text { IR image of } \\
\text { knife gate }\end{array}$} & 1 & 159 & 0.6692 & 27.6544 & 145 & 0.5083 & 27.7361 \\
\hline & 2 & 73,160 & 0.7483 & 30.4519 & 73,160 & 0.5901 & 30.4519 \\
\hline & 3 & $20,87,160$ & 0.8019 & 30.5362 & $22,97,160$ & 0.6706 & 32.4381 \\
\hline & 4 & $20,86,135,183$ & 1.3096 & 30.9541 & $20,87,160,202$ & 0.8835 & 31.6502 \\
\hline
\end{tabular}

From the above results, the multilevel threshold segmentation method with two-dimensional entropy based on bat algorithm is superior to the method based on PSO algorithm in time consuming and segmentation accuracy.

\section{Conclusions}

Aiming at the difficulty of thresholds selection and the problem of time consuming during the process of intelligent diagnosis of power equipment, an IR image segmentation method with multilevel threshold of two-dimensional entropy based on bat algorithm is proposed. By introducing the bat algorithm, the multilevel threshold segmentation of the IR image temperature field is accurately and effectively. In the experiment, the multilevel threshold segmentation method with two-dimensional entropy based on bat algorithm or PSO algorithm is used to segment the IR images. The results show that the time consuming of this method is decreased by $20.92 \%, 21.15 \%, 15.92 \%$ and $32.27 \%$ in single-threshold, double-threshold, triple-threshold and quadruple-threshold segmentation method, and the accuracy of segmentation is higher than that of two-dimensional entropy segmentation method based on PSO algorithm. Therefore, the proposed method can solve the multilevel threshold segmentation problem of IR image of power equipment quickly and effectively.

\section{Acknowledgements}

This work was supported by the NSFC (61107081, 11647023), Shanghai Local Colleges and Universities capacity Building Program (15110500900), Shanghai Natural Science Foundation, China (17ZR1411500), and Intelligent Power Grid Center Knowledge Service Platform Achievement Cultivation Project (A-0010-16-029-001).

\section{References}

[1] Cui Haoyang, Xu Yongpeng, Sun Yue, et al. Substation Infrared Image Fuzzy Enhancement Algorithms Based on Improved Adaptive Genetic Theory [J]. High Voltage Engineering, 2015, 41(3): 902-908. 
[2] Men Hong, Yu Jiaxue, Qin Lei. Segmentation of Electric Equipment Infrared Image Based on CA and OTSU [J]. Electric Power Automation Equipment, 2011, 31(9): 92-95.

[3] Chen Fang, Yao Jiangang, Li Zuosheng, et al. The Method to Extract Shed Surface Image of a Single Insulator From Infrared Image of an Insulator String [J]. Power System Technology, 2010, 34(5): 220-224.

[4] Chen Heng, Hou Shanjing. Fault Diagnosis for Electrical Equipment Using Infrared Thermography [M]. Beijing: China Electric Power Press, 1998.

[5] Zou Hui, Huang Fuzhen. Infrared Image Segmentation for Electrical Equipment Based on FAsT-Match Algorithm [J]. Infrared Technology, 2016, 38(1): 21-27.

[6] Wang Qiyin, Xue Jiandong, Ren Xinhui. An Adaptive Segmentation Method of Substation Equipment Infrared Image [J]. Infrared Technology, 2016, 38(9): 770-773.

[7] Liu Yitong, Fu Mengyin. A Fast Infrared Image Segmentation Method Based Two-Dimensional Entropy and Particle Swarm Optimization on Algorithm [J]. Pattern Recognition and Artificial Intelligence, 2008, 21(2): 155-159.

[8] Chen Kai, Chen Fang, Dai Min, et al. Fast Image Segmentation with Multilevel Threshold of Two-dimensional Entropy Based on Firefly Algorithm [J]. Optics and Precision Engineering, 2014, 22(2): 517-523.

[9] Wang Yahui, Jia Chenhui, Zhao Renpeng. Multi-objective Bat Algorithm Based on Decomposition [J]. Transactions of the Chinese Society for Agricultural Machinery, 2015, 46(4): 316-324.

[10]Fu Jiacai, Lu Qingsong. Fault Sections Location of Distribution Network Based on Bat Algorithm [J]. Power System Protection and Control, 2015, 43(16): 100-105.

[11]Liu Changping, Ye Chunming. Bat Algorithm with Chaotic Search Strategy and Analysis of Its Property [J]. Journal of System Simulation, 2013, 25(6): 1183-1188, 1195. 